\title{
Topologically ordered zigzag nanoribbon: $e / 2$ fractional edge charge, spin-charge separation, and ground-state degeneracy
}

\author{
S.-R. Eric Yang, ${ }^{1, *}$ Min-Chul Cha $\odot,{ }^{2}$ Hye Jeong Lee, ${ }^{1}$ and Young Heon Kim ${ }^{1}$ \\ ${ }^{1}$ Department of Physics, Korea University, Seoul 02855, Korea \\ ${ }^{2}$ Department of Photonics and Nanoelectronics, Hanyang University, Ansan 15588, Korea
}

(Received 26 January 2020; accepted 29 June 2020; published 21 July 2020)

\begin{abstract}
We numerically compute the density of states (DOS) of interacting disordered zigzag graphene nanoribbon (ZGNR) having midgap states showing $e / 2$ fractional edge charges. The computed Hartree-Fock DOS is linear at the critical disorder strength where the gap vanishes. This implies an $I-V$ curve of $I \propto V^{2}$. Thus, $I$ - $V$ curve measurement may yield evidence of fractional charges in interacting disordered ZGNR. We show that even a weak disorder potential acts as a singular perturbation on zigzag edge electronic states, producing drastic changes in the energy spectrum. Spin-charge separation and fractional charges play a key role in the reconstruction of edge antiferromagnetism. Our results show that an interacting disordered ZGNR is a topologically ordered Mott-Anderson insulator.
\end{abstract}

DOI: 10.1103/PhysRevResearch.2.033109

\section{INTRODUCTION}

Graphene has numerous remarkable properties [1-3]. One prominent feature is that, in the absence of disorder, zigzag graphene nanoribbons (ZGNRs) can support chiral symmetry protected topological (SPT) [4-9] edge states displaying an integer charge [10]. Disorder has profound effects on ZGNRs. In particular, an interacting disordered ZGNR becomes a Mott-Anderson insulator [11-13] with spin-split energy levels [14]. Furthermore, localized gap-edge states reduce the size of the gap between the occupied and unoccupied midgap states with energies $-\Delta_{S} / 2$ and $\Delta_{S} / 2$, respectively, to $\Delta_{S}$ (see Fig. 1). In the weak disorder regime, solitonic midgap states $[15,16]$ may have an $e / 2$ fractional charge on each of the opposite zigzag edges, i.e., there is one for each edge [14], where $e$ is the electron charge, see Fig. 2. These fractional charges have small disorder-induced charge variances. In addition, the charge fractionalization is protected against quantum charge fluctuations by the nonzero $\Delta_{s}$. Here $\Delta_{s} \lesssim 10^{-2} \Delta \sim 1 \mathrm{THz}$, where $\Delta$ is the gap value; this is sufficiently large that quantum charge fluctuations can be ignored (see Girvin [17]). In the absence of disorder, typically, $\Delta \sim 0.2 t$ [18], where $t \sim 3 \mathrm{eV}$ is the hopping constant.

An excellent opportunity to observe these boundary charges has recently arisen, as rapid progress has been made in the fabrication of atomically precise GNRs [19]. The chiral Luttinger liquid theory of fractional quantum Hall edges [20,21] predicts an $I-V$ curve of $I \propto V^{1 / \nu}$. The

\footnotetext{
*Corresponding author: eyang812@gmail.com
}

Published by the American Physical Society under the terms of the Creative Commons Attribution 4.0 International license. Further distribution of this work must maintain attribution to the author(s) and the published article's title, journal citation, and DOI. corresponding DOS is given by

$$
\rho(E) \propto E^{1 / \nu-1},
$$

where $v$ is the filling factor, and the energy $E$ is determined from the Fermi energy (these edges support gapless excitations). This predicted $I-V$ curve has been experimentally confirmed [22]. It should be noted that Laughlin quasiparticles have an odd denominator fractional charge $e \nu$, and an even denominator fractional charge $e / 2$ is not found in fractional quantum Hall systems. The aforementioned $I-V$ curve may be derived heuristically by assuming that a tunneling electron fractionalizes into $m=1 / v$ fractionally charged quasiparticles [23], where

$$
e \rightarrow e / m+\cdots+e / m \text {. }
$$

(This tunneling process is illustrated in Fig. 3.) However, the chiral Luttinger liquid theory does not apply to ZGNRs. Furthermore, the gap-edge states are all localized along the ribbon direction, in contrast to the fractional quantum Hall edge states. Moreover, the average edge charge of the gapedge states with energies within a small interval $\delta E$ is $e / 2$; however, significant disorder-induced charge fluctuations may occur. Some of these states are more localized on the left or right zigzag edges. This tendency increases as the electron energy deviates from $\pm \Delta_{s} / 2$. Despite this, if we apply the above heuristic argument to a ZGNR with $m=2$, then we find that the $I-V$ curve is given by

$$
I \propto \int d \epsilon_{1} \int d \epsilon_{2} \theta\left(e V-\epsilon_{1}-\epsilon_{2}\right) \propto V^{2},
$$

where $\theta$ and $\epsilon_{1,2}$ are the step function and quasiparticle energies, respectively. This $I-V$ curve is equivalent to a linear tunneling DOS. A topological insulator is usually not significantly affected by a disorder potential, but the SPT phase of a ZGNR is profoundly changed by disorder. However, the 
(a)

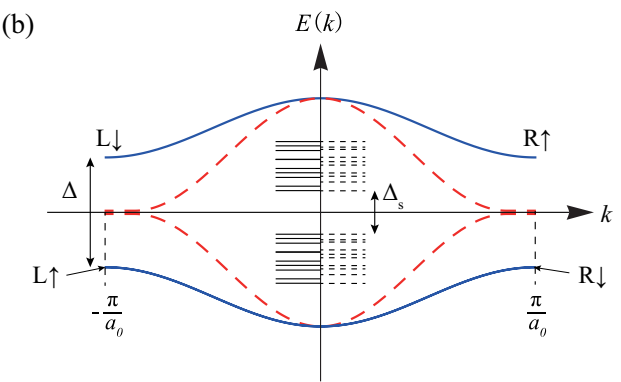

FIG. 1. (a) Zigzag edge antiferromagnetism of interacting ZGNR without disorder. (b) Schematic of interacting (solid curves) and noninteracting (dashed curves) ZGNR band structures. The unoccupied and occupied states near the wave vectors $k= \pm \pi / a_{0}$ are shown: $R$ and $L$ represent states confined on the right and left zigzag edges, respectively ( $a_{0}=1.73 a$ is the unit cell length of the ZGNR and $a=1.42 \AA$ is the $\mathrm{C}$-C distance). The small arrows indicate spins. The spin-split energy levels of the spin-up (solid lines) and spin-down (dashed lines) gap-edge states of the interacting disordered ZGNRs are shown. These states decay exponentially from the zigzag edges. In the limit of an infinitely long ribbon the gap $\Delta_{s}$ may vanish and a soft gap can develop. Another degenerate ground state can be obtained by exchanging $\uparrow$ and $\downarrow$ spins.

physical processes involved in this effect and the properties of the interacting disordered state are not well understood.

In this study, we propose an experiment that may provide evidence of the presence of $e / 2$ fractional charges in interacting disordered ZGNRs. We compute the DOS of an interacting disordered ZGNR and find that, for the critical disorder strength where the ZGNR supports gapless excitations (i.e., where $\Delta_{s}$ vanishes), our computed Hartree-Fock (HF) DOS is linear near the Fermi energy. This finding is in agreement with the heuristic argument given above. In addition, our results show that even a weak disorder potential behaves similar to a singular perturbation on zigzag edge electronic states, generating drastic changes in the energy spectrum. It also induces a magnetic zigzag edge reconstruction in which fractional edge charges and spin-charge separation play a significant role. Moreover, disorder also changes an SPT phase to a topologically ordered phase [5,24].

\section{MODEL}

There are two types of disorder, namely diagonal and off-diagonal disorder. We model off-diagonal disorder by randomly varying the nearest-neighbor hopping parameters,

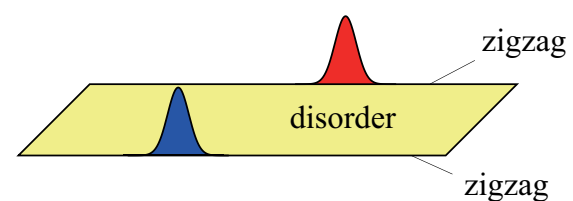

FIG. 2. Two $e / 2$ fractional zigzag edge charges of an interacting disordered ZGNR. Note that red (blue) probability density means that the wave function has A (B) chirality, i.e., it is finite only on A (B) carbon atoms. Since there is negligible tunneling between these sites we will call this type of state a mixed chiral state.

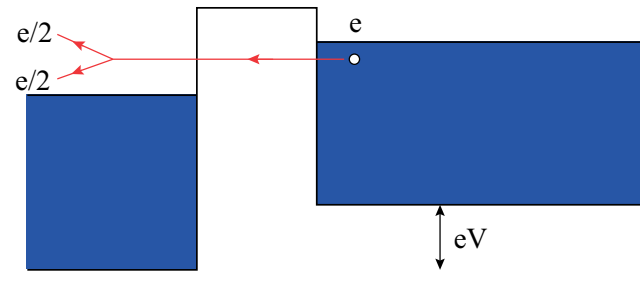

FIG. 3. A tunneling electron is fractionalized when it enters an interacting disordered ZGNR.

see Fig. 4. However, since the results of off-diagonal disorder and diagonal disorder are similar we mainly report, in this study, on the results of diagonal disorder, shown schematically in Fig. 5. In diagonal disorder $N_{\text {imp }}$ defects or short-ranged impurities are randomly placed at carbon sites $\vec{R}_{j}$. Let us analyze the scattering of left and right edge states by a short-ranged disorder potential. Consider a spin-up electron at $k=\frac{\pi}{a_{0}}$ with the wave function $\phi_{R \uparrow}$ localized on the right zigzag edge. For a short-ranged potential, a significant wave vector transfer in a backscattering occurs for $\left|k-k^{\prime}\right| \sim 1 / a_{0}$ [25]. Such a short-ranged disorder potential couples the chiral zigzag edge state $R \uparrow$ to another chiral zigzag edge state $L \uparrow$ on the opposite zigzag edge at $k=-\frac{\pi}{a_{0}}$, as shown in Fig. 6 [their wave functions $\phi_{R}$ and $\phi_{L}$ are depicted in Figs. 7(a) and 7(b)]. This process produces the bonding or antibonding edge state with the wave function $\frac{1}{\sqrt{2}}\left(\phi_{L}+\phi_{R}\right)$ or $\frac{1}{\sqrt{2}}\left(\phi_{L}-\phi_{R}\right)$. The probability density of one of these states is shown schematically in Fig. 7(c) (a mixed chiral state). These states display charge fractionalization with $1 / 2$ charges on the left and right zigzag edges. But states with uneven fractions may also be generated. Numerical calculations are needed to determine the distribution of these edge charges.

The strength of the potential $\epsilon_{j}$ is chosen randomly from the energy interval $[-\Gamma, \Gamma]$. The values of $\epsilon_{j}$ depend on the type of charged impurities in the substrate and defects in graphene. The number of impurities or defects is also relevant in determining the strength of the disorder potential. In the Born approximation disorder strength is characterized by the parameter $\Gamma \sqrt{n_{\mathrm{imp}}}$, where $n_{\mathrm{imp}}=N_{\mathrm{imp}} / N$ is the ratio between

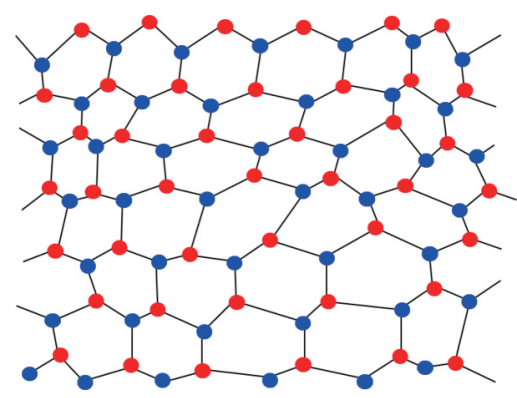

FIG. 4. Off-diagonal disorder: Random network of hexagons consisting of A and B carbon atoms. A zigzag edge site is connected to two other carbon atoms while a site away from the edges is connected to three other carbon atoms. The hopping parameter $t$ is not the same for all sites. The zigzag edges have definite chirality, consisting $\mathrm{A}$ or $\mathrm{B}$ carbon atoms. In contrast, armchair edges have mixed chirality. 


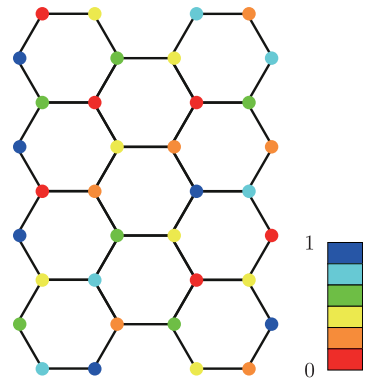

FIG. 5. Diagonal disorder: Site energies are varied randomly. Colors represent strength of on-site disorder potential. Again a zigzag edge site is connected to two other carbon atoms while a site away from the edges is connected to three other carbon atoms. But the hopping parameter $t$ is the same for all sites.

the numbers of impurities and total carbon atoms. The range of an impurity potential is important in determining properties of the Dirac electrons in ZGNRs [25-27]. However, a shortranged disorder potential gives more robust charge fractionalization [14]. In the following all our numerical results are for short-ranged potentials, unless stated otherwise.

We applied a Hubbard model to the interacting disordered ZGNRs and used a self-consistent HF approximation (HFA); this is because the self-consistency provides an excellent approximation when both disorder and interactions are present [28-30]. We include both electron-electron interactions and disorder in a tight-binding model at half-filling. When $U=0$ disorder can be treated exactly in the HFA while in the other limit, where disorder is absent, interaction effects can be represented well by the HFA, which widely used in graphene-related systems [31,32]. Its results are consistent with those of density-functional theory [18]. The total Hamiltonian in the HFA is

$$
\begin{aligned}
H= & -\sum_{\langle i j\rangle \sigma} t_{i j} c_{i \sigma}^{\dagger} c_{j \sigma}+\sum_{i \sigma} \epsilon_{i} c_{i \sigma}^{\dagger} c_{i \sigma} \\
& +U \sum_{i}\left(n_{i \uparrow}\left\langle n_{i \downarrow}\right\rangle+\left\langle n_{i \uparrow}\right\rangle n_{i \downarrow}-\left\langle n_{i \uparrow}\right\rangle\left\langle n_{i \downarrow}\right\rangle\right) \\
& -\frac{U}{2} \sum_{i}\left(n_{i \uparrow}+n_{i \downarrow}\right),
\end{aligned}
$$

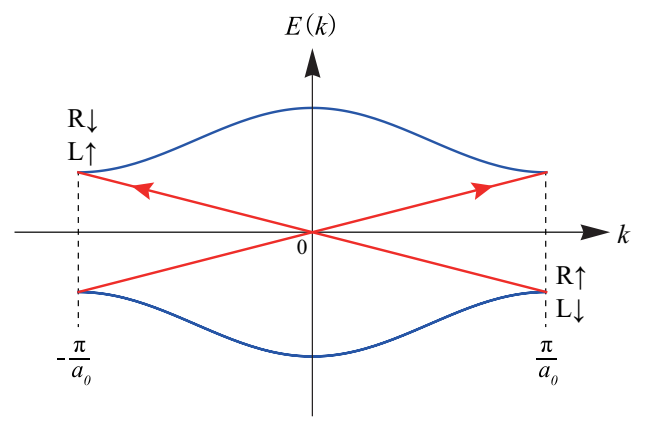

FIG. 6. States localized on the right and left zigzag edges are represented, respectively, by $R$ and $L$. The long arrows indicate the coupling, induced by a short-ranged disorder potential, between states $R \uparrow$ and $L \uparrow$ or $R \downarrow$ and $L \downarrow$. (a)

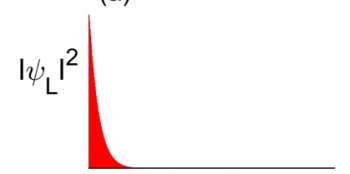

(c)

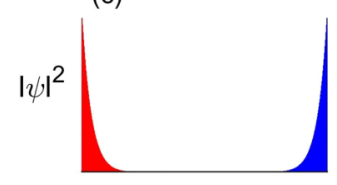

FIG. 7. Schematic drawing of the site probability distribution $|\psi|^{2}$ of two degenerate edge states with wave functions $\psi_{L}$ and $\psi_{R}$ is shown. They are localized on the (a) left and (b) right edges, respectively. (c) Disorder couples these states and can generate antibonding and bonding edge states with the wave functions $\psi=\frac{1}{\sqrt{2}}\left(\phi_{L}-\phi_{R}\right)$ and $\psi=\frac{1}{\sqrt{2}}\left(\phi_{L}+\phi_{R}\right)$, respectively.

where $c_{i \sigma}^{\dagger}$ and $n_{i \sigma}$ are the electron creation and occupation operators at site $i$ with spin $\sigma$. Since the translational symmetry is broken, the Hamiltonian is written in the site representation. In the hopping term the summation is over the nearestneighbor sites (the average value of hopping parameters is $\left.\left\langle t_{i j}\right\rangle=t \sim 3 \mathrm{eV}\right)$. The eigenstates and eigenenergies are computed numerically by solving the tight-binding Hamiltonian matrix self-consistently. The self-consistent occupation numbers $\left\langle n_{i \sigma}\right\rangle$ in the Hamiltonian are the sum of the probabilities to find electrons of $\operatorname{spin} \sigma$ at site $i$ :

$$
\left\langle n_{i \sigma}\right\rangle=\sum_{E \leqslant E_{F}}\left|\psi_{i \sigma}(E)\right|^{2} .
$$

The sum is over the occupied eigenstates with energy $E$ below the Fermi energy $E_{F}$. Note that $\left\{\psi_{i \sigma}(E)\right\}$ represents an eigenvector of the tight-binding Hamiltonian matrix with energy $E$. For notational simplicity, we suppress its dependence on $E$ from now on. The ratio between the disorder strength and interaction strength is $\kappa=\Gamma \sqrt{n_{\text {imp }}} / U \ll 1$ in the weak disorder regime. Varying the strength $\Gamma$ is approximately equivalent to changing $\sqrt{n_{\text {imp }}}$. In this work, the ribbon width was set to $w=7.1 \AA$ and the on-site repulsion was $U=t$. To investigate very long ZGNRs it is vital to use sparse matrix diagonalization techniques.

\section{QUANTIZED FRACTIONAL CHARGE OF MIDGAP STATE}

For the sake of clarity we briefly summarize the results we obtained in Ref. [14]. The midgap states with energy $|E| \approx \Delta_{S} / 2 \ll \Delta / 2$ and edge charge $q_{A} \approx 1 / 2$ represent soliton states. They consist of almost equal contributions from the valence $R$ and conduction band $L$ states (or from the valence band $L$ and conduction band $R$ states) with energies near $-\Delta / 2$ and $\Delta / 2$, respectively, as shown in Fig. 1. A soliton state has small disorder-induced charge fluctuations. In addition, spin-split states are also present [8], as in a MottAnderson insulator [13]. For a given disorder realization, greater spin-splitting occurs for states with $|E| \approx \Delta_{S} / 2$ than for those with $|E| \approx \Delta / 2$. In the limit where disorder strength 

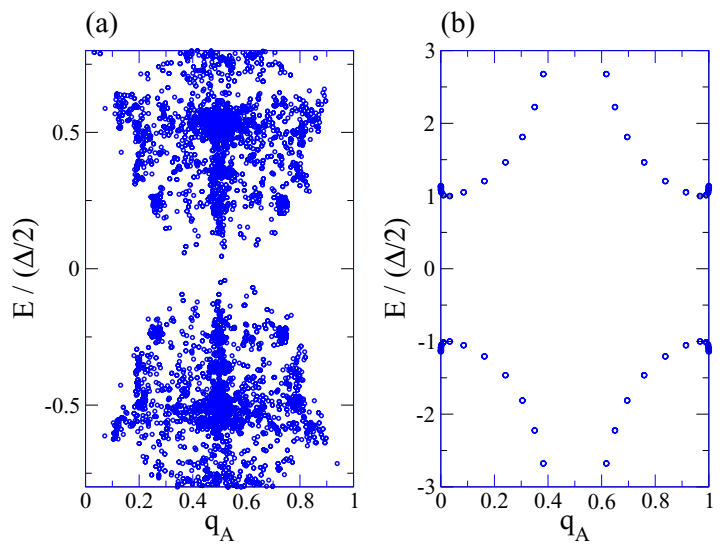

FIG. 8. (a) Plot of $q_{A}$ for disorder potential strength $\Gamma=0.1 t$, where each point represents the probability of finding an electron of a gap-edge state on $A$ carbon sites, $q_{A}$. The disorder realization number is $N_{D}=300$, and the ribbon length is $\ell=196.8 \AA$. Here the impurity or defect-to-carbon atom ratio is $n_{\text {imp }}=0.1$. The gap size is $\Delta_{s} \approx$ $0.12 \frac{\Delta}{2}$. (b) Plot of $q_{A}$ for $U=t$ in the absence of disorder, where the on-site electron repulsion and hopping parameter are indicated by $U$ and $t$, respectively.

$\Gamma \rightarrow 0$ and ribbon length $\ell \rightarrow \infty$ the energy of a soliton decreases toward $E=0$ and $q_{A} \rightarrow 1 / 2$ with very small fluctuations, i.e., the value of the fractional charge approaches $e / 2$.

Here we provide a new and efficient way to analyze numerical results. The numerical results are presented in the following way: For each HF quasiparticle state with energy $E$ and spin $\sigma$ we compute the total probability density on A carbon sites, denoted by

$$
q_{A}=\sum_{i \in A}\left|\psi_{i \sigma}(E)\right|^{2}
$$

We plot all the possible values of $\left(E, q_{A}\right)$. This plot makes it easier to delineate physics behind charge fractionalization. We find that disorder behaves similar to a singular perturbation on zigzag edge electronic states [33]. This singular perturbation is analogous to the nonperturbative coupling between the left and right wells of a double quantum well (the nonperturbative aspect can be seen by using instantons of the inverted double well potential [34]). A disorder potential or a magnetic field can produce drastic changes in the electron wave functions [35,36], see Figs. 8(a) and 8(b). Figure 8(a) shows the distribution of $\left(E, q_{A}\right)$ for $\Gamma=0.1 t$. Note that particle-hole symmetry (chiral symmetry) is broken. Even a weaker disorder potential with $\Gamma=0.03 t$ produces similar drastic changes in the energy spectrum when compared to the disorder-free behavior, see Fig. 9. Note that in this weak disorder regime charge fractionalization is more accurate: Midgap states shown in the figure has $q_{A}$ very close to $1 / 2$. In contrast to the case of $\Gamma=0$, shown in Fig. 8(b), there are numerous states with $q_{A} \approx 1 / 2$ in the energy range $|E|<$ $\Delta / 2$. If the disorder potential experienced by the left and right edges differs, then charge values will deviate from $1 / 2$.

We have also examined off-diagonal disorder. In the presence of off-diagonal disorder, a zigzag edge site is connected to two other carbon atoms while a site away from the edges are connected to three other carbon atoms, but the hopping

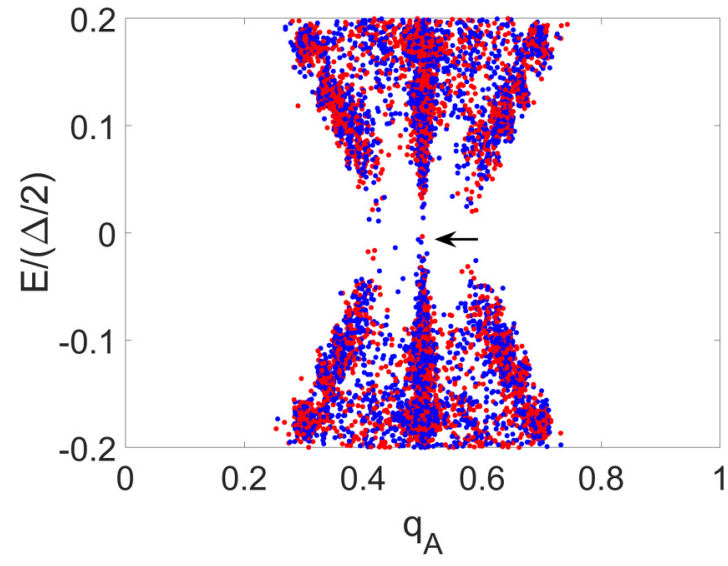

FIG. 9. Plot of $q_{A}$ for $\Gamma=0.03 t, U=t, n_{\text {imp }}=0.1, \quad l=$ $1232.5 \AA$, and $N_{D}=2166$. Zero-energy states with $q_{A}$ rather close to $1 / 2$ are indicated by an arrow. Blue (red) dots are for spin-up (-down) states.

parameter is not the same for every site. Despite this our numerical results show that fractional midgap states do exist as in diagonal disorder, see Figs. 10 and 11. The network topology, i.e., how many carbon atoms each site of the zigzag edges is connected to, is crucial for charge fractionalization. Whether disorder is off-diagonal or diagonal is immaterial.

We find that the localization length along the edges decreases as $|E|$ decreases toward $\Delta_{s} / 2$. A small localization length means that the repulsive energy between an electron in a soliton state and an added electron in another soliton state can be small since they can avoid each other. This effect determines the magnitude of $\Delta_{s}$.

\section{LINEAR TUNNELING DENSITY STATES}

The $e / 2$ fractional charge fluctuations decrease as $|E| \rightarrow$ $\Delta_{s} / 2$. Thus, we investigated the effect of this behavior on the DOS near the Fermi energy. (Note that the tunneling DOS measures the number of quasiparticle excitations of the interacting disordered ZGNR.) We examined longer ZGNRs than those in Ref. [14]. This allows us to extract the behavior of the DOS in the limit $E \rightarrow 0$. We performed finite-size

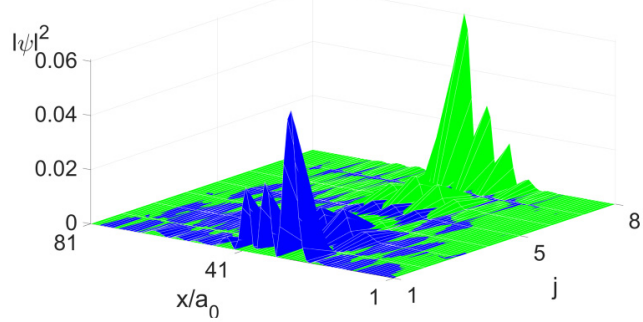

FIG. 10. Plot of the probability density of a fractionalized gapedge state in the presence of off-diagonal disorder. It is a mixed chiral state; green and blue represent different chiralities. Its energy is $E=-0.019 t$ with $q_{A}=0.495$. The range of hopping parameters is $0.94<t_{i j} / t<1.06$ and the ribbon length is $\ell=199.3 \AA$. A zigzag ribbon consists of zigzag lines. In this ribbon they are labeled from $j=1$ to 8 . 


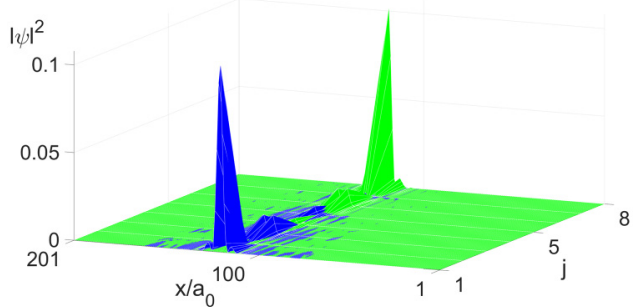

FIG. 11. Same as in Fig. 10 but with a longer ribbon length $\ell=$ $494.5 \AA$. The gap-edge-state energy is $E=0.055 t$ with $q_{A}=0.51$. The range of hopping parameters is $0.94<t_{i j} / t<1.06$.

calculations and computed the DOS given by

$$
\rho(E)=\frac{D_{\delta E}(E)}{l N_{D} \delta E},
$$

where $D_{\delta E}(E)$ is the total number of states in the energy histogram interval $\delta E$ and $N_{D}$ is the number of disorder realizations. We defined the critical point $\Gamma_{c}$ as the value where $\Delta_{s}$ is zero, i.e., where the gap closes. The heuristic argument given above suggests that the DOS at $\Gamma_{c}$ is linear near the Fermi energy. The DOS result for $\Gamma=0.18 t \gtrsim \Gamma_{c}$ is plotted in Fig. 12. Our numerical results show that the energy range where the DOS is linear increases as ribbon length $\ell$ grows and that fluctuations in the DOS also decreases. Note that $\Gamma_{c}$ does not represent a metal-insulator transition point, and the gap-edge states are all localized in the interacting disordered ZGNR [37]. Note also that $\Gamma_{c}$ decreases as $\ell$ increases (this is a finite-size effect).

In the limit $\ell \rightarrow \infty$ our results for $\Gamma<\Gamma_{c}$ suggest that the DOS decreases exponentially to zero as $E \rightarrow 0$, see Fig. 13 . The shape of the resulting soft gap can be fitted well with an exponential form of

$$
\rho(E)=A\left(e^{\alpha x^{2}}-1\right),
$$

where $x=E /(\Delta / 2)$ and the fitting parameters are $A=0.164$ and $\alpha=162$. This suggests that the size of the exponential gap is of the order of $\sim 0.05 \frac{\Delta}{2}$.

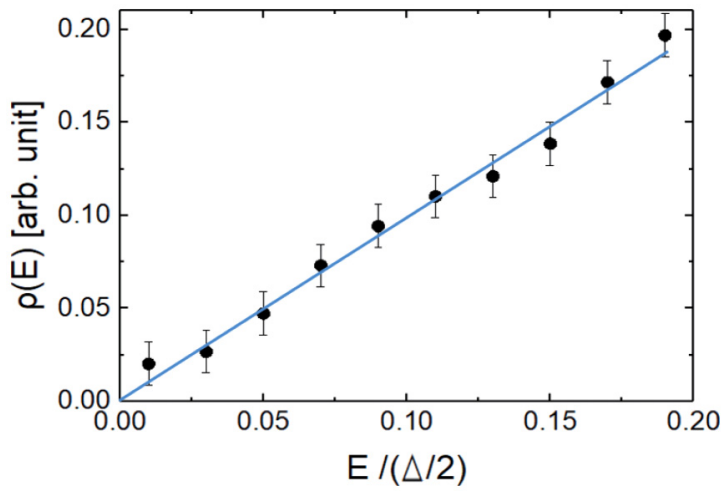

FIG. 12. Plot of DOS $\rho(E)$ for disorder potential strength $\Gamma=$ $0.18 t$. Here the disorder realization number $N_{D}=4409$, the impurity or defect-to-carbon atom ratio $n_{\text {imp }}=0.1$, and ribbon length $\ell=$ $740.46 \AA$. The histogram interval is $\delta E=0.02 \frac{\Delta}{2}$. The solid line represents a linear fit to $\rho(E)$.

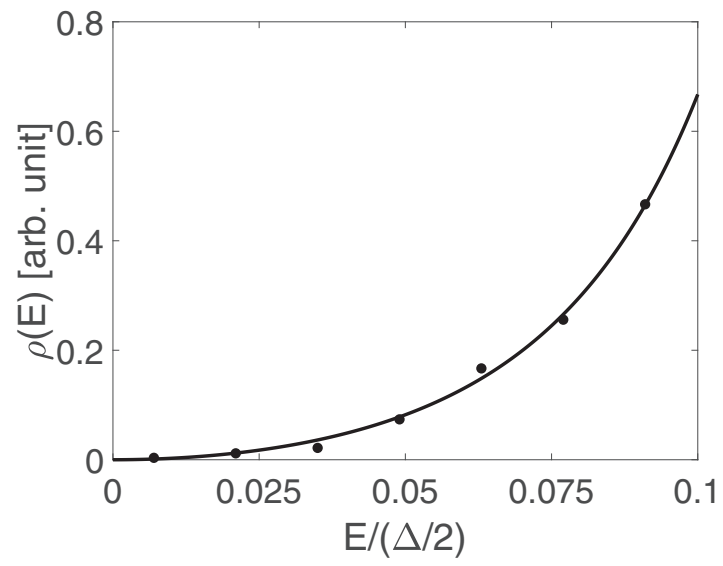

FIG. 13. The DOS displays an exponential soft gap near $E=0$. Parameters are $U=t, \Gamma=0.03 t, n_{\text {imp }}=0.1, l=1232.5 \AA$, and $N_{D}=2166$. Histogram interval is $0.014 \frac{\Delta}{2}$.

\section{SPIN-CHARGE SEPARATION}

An interacting disordered ZGNR displays antiferromagnetism that is weakly perturbed, see Fig. 14. As shown in Fig. 1(a) a zigzag ribbon consists of zigzag lines. Away from the outer two zigzag edges a zigzag line inside the ribbon is mostly antiferromagnetically coupled with the neighboring two zigzag lines. Magnetization is mostly ferromagnetic on each of the two boundary zigzag edges but the two zigzag edges are antiferromagnetically coupled. On the left zigzag edge the site spin direction flips in a region. Also note that on the right zigzag edge the site spin values are nearly zero in two regions. These effects are due to the singular nature of disorder, as we explain below.

Let us introduce disorder into the pure SPT phase and try to understand how the singular disorder potential disrupts the SPT phase. Suppose that the site occupation numbers of the disorder-free left edge are $n_{i \uparrow}=0.7$ and $n_{i \downarrow}=0.3$. Then those of the right edge are $n_{i \uparrow}=0.3$ and $n_{i \downarrow}=0.7$, respectively.

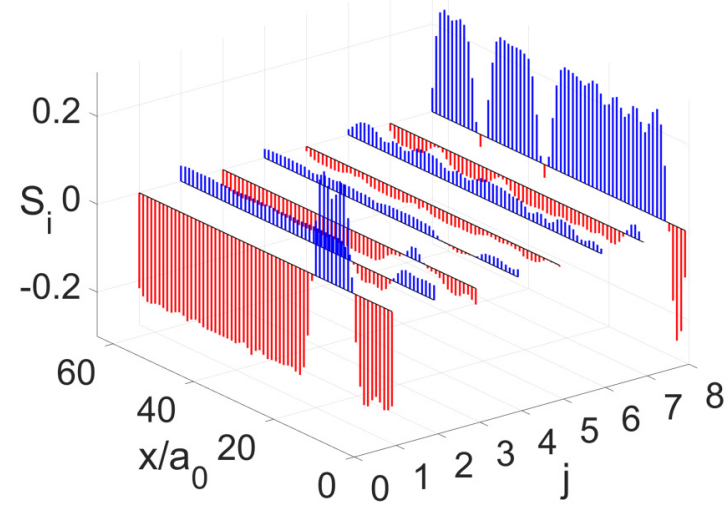

FIG. 14. Net site spin values $S_{i}=S_{i \uparrow}+S_{i \downarrow}=n_{i \uparrow}-n_{i \downarrow}$ are plotted, where $n_{i \sigma}$ is the site occupation number for spin $\sigma$. A zigzag ribbon consists of zigzag lines. In this ribbon they are labeled from $j=1$ to 8 . Blue (red) lines indicate positive (negative) spin values. This result is for one disorder realization with diagonal disorder. The parameters are $U=t$. 


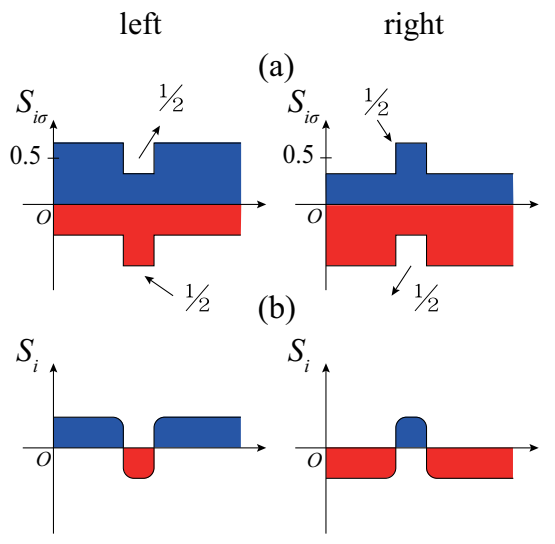

FIG. 15. (a) Site-spin $z$ components, $S_{i \uparrow}=n_{i \uparrow}$ (blue) and $S_{i \downarrow}=$ $-n_{i \downarrow}$ (red), plotted along edges. Here $n_{i \sigma}$ is the site occupation number. The left and right figures correspond to the left and right zigzag edges, respectively. The number $1 / 2$ indicates a removed or added electron occupation number. (b) Net site spin values $S_{i}=$ $S_{i \uparrow}+S_{i \downarrow}$ are plotted.

Assume that disorder generates one spin-up and one spindown occupied soliton state near the gap edge displaying charge fractionalization. In other words, a spin-up electron on the left zigzag edge of the interacting ZGNR is converted into two $e / 2$ fractional charges, one of which resides on the left zigzag edge while the other resides on the right zigzag edge [Fig. 15(a)]. Similarly, a spin-down electron on the right zigzag edge is also replaced by two $e / 2$ fractional charges, with one each residing on the left and right zigzag edges [Fig. 15(a)]. Hence, the total $\mathrm{z}$ component of the site spin $S_{i}=S_{i \uparrow}+S_{i \downarrow}$ on the zigzag edges changes sign along the edge direction, as shown in Fig. 15(b). The total occupation number of each site $n_{i}=n_{i \uparrow}+n_{i \downarrow}$ is now close to one (i.e., the ZGNR is half-filled). Note that the disorder potential creates an even number of solitons to minimize the energy cost of double occupancy of a site (a soliton consists a pair of fractional charges). Thus, even if the disorder potential is weak it can still disrupt the SPT phase. In addition, the magnetic zigzag edge reconstruction can also lead to a spincharge separation $[15,23,38]$. Figures $16(a)$ and $16(b)$ show how a charge fractionalization process results in an object
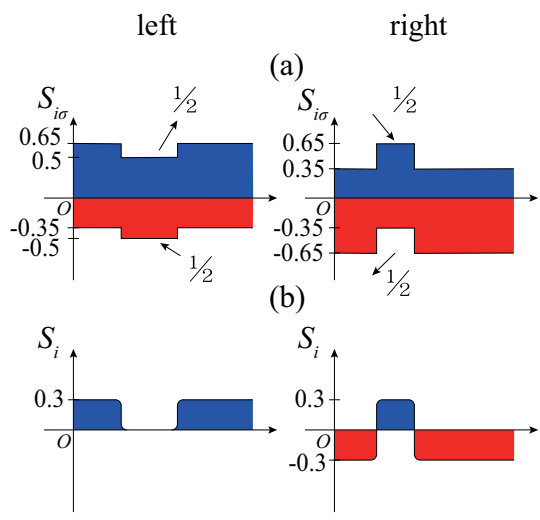

FIG. 16. Plot of total $z$ component of ground-state site spin $S_{i}$. Process of spin-charge separation is displayed in (a) and (b).
$\left(e_{L}, 0\right)$ that displays spin-charge separation. Here $e_{L}$ denotes an electron charge located on the left edge and number 0 means no spin. When such an object moves along the zigzag edge it will carry charge but no spin.

\section{MAIN PHYSICS}

Disorder has profound effects on a ZGNR as it breaks particle-hole symmetry (chiral symmetry), in addition to inducing spin splitting. Other symmetries are also broken: translational, reflection, rotational, and inversion symmetries. Spin-rotational symmetry is spontaneously broken [39]. Time-reversal symmetry is already broken by antiferromagnetism. There is no symmetry that will protect edge states in an interacting disordered ZGNR. Moreover, as we already remarked, there are doubly degenerate ground states that are connected to each other via reversal of electron spin directions (see Fig. 1). All this suggests that an interacting disordered ZGNR is qualitatively different from the disorder-free interacting ZGNR.

We now explain the essential physics of charge fractionalization and the physical nature of interacting disordered ZGNRs. The $e / 2$ charges are a result of the subtle interplay between network topology of the underlying lattice, electron correlation and disorder. In each disorder realization particlehole symmetry (chiral symmetry) is broken, but after disorder averaging the symmetry is approximately restored. This implies that the average edge charge is $e / 2$ at each energy $|E|<\Delta / 2$, but with a significant charge variance. However, if the tunneling DOS develops a soft gap [28,40], then the charge variance near zero energy will be negligible in the weak disorder regime, see Fig. 9. What is the physical origin of a soft gap? The essential physics is that it is difficult for the tunneling electron to avoid other electrons since it takes long time for interacting electrons to diffuse away from each other (see Girvin and Yang, Ref. [23], pp. 290 and 645). Our numerical simulation shows that an interacting disordered ZGNR cannot be reached iteratively from a disorder-free chiral SPT state. Moreover, an interacting disordered ZGNR has a doubly degenerate ground state, $e / 2$ fractional charges, spin-charge separation, and broken chiral symmetry. Thus we expect that it is in a topological ordered phase rather than in an SPT phase (see Wen [5] for the distinction between them). An interacting disordered ZGNR is somewhat analogous to topologically ordered Laughlin states. In both systems fractional charge and ground-state degeneracy are intimately related $[5,23]$.

\section{CONCLUSIONS}

In conclusion, an interacting disordered ZGNR is a onedimensional topologically ordered insulator with $e / 2$ solitonic fractional charges and with twofold ground-state degeneracy. Even a weak disorder potential behaves similar to a singular perturbation, producing spin-splitting and drastically modifying the energy spectrum. We conducted a numerical study showing that the DOS is linear at the critical disorder strength. Measurement of the $I-V$ curve may thus provide evidence for the presence of fractional charges in an interacting disordered ZGNR. We also found that spin-charge separation and fractional edge charges play a significant role in the 
reduction of edge antiferromagnetism. We hope that our work will stimulate experimental tests investigating the presence of $e / 2$ fractional charges in interacting disordered ZGNRs. However, several experimental possibilities and challenges exist. In particular, investigation of tunneling between zigzag edges, as in fractional quantum Hall bar systems [41], may be fruitful. Quantum shot noise may directly measure [42] the tunneling fractional charge of a ZGNR. Resonant tunneling measurement through a quantum dot structure made of a rectangular ZGNR may also be explored [43]. Finally, it would be interesting to investigate other zigzag nanoribbon systems that exhibit antiferromagnetism, e.g., silicene and boron nitride nanoribbons $[44,45]$. Disorder can couple the left and right zigzag edges and lead to charge fractionalization.

\section{ACKNOWLEDGMENTS}

This research was supported by the Basic Science Research Program through the National Research Foundation of Korea (NRF), funded by the Ministry of Education, ICT, and Future Planning (MSIP) [NRF-2018R1D1A1A09082332 (S.-R.E.Y.) and NRF-2019R1F1A1062704 (M.-C.C.)].

\section{APPENDIX: PARTICLE FRACTIONALIZATION IN OTHER SYSTEMS}

Kitaev's chain [46], polyacetylene, and interacting disordered ZGNRs all have end states. There are similarities and differences between them [16]. It is both interesting and instructive to take note of them.

Chiral symmetry guarantees existence of edge states in ZGNRs and polyacetylene. However, in the presence of disorder there is an important difference between ZGNRs and polyacetylene. Let us consider the Su-Schrieffer-Heeger effect in detail [47]. Consider finite-length polyacetylene in one of the dimerized phases, see Fig. 17. The electron density is uniform with occupation number $n_{i}=1$ at all sites $i$. Here two nearly degenerate soliton end states exist (bonding and antibonding states); one will be occupied and the other unoccupied. Disorder will spilt these states because their wave functions are somewhat different. Then two possibilities are present. (a) The boundary fractional charges will suffer quantum charge fluctuations because the energy splitting is small (only a large energy splitting will suppress quantum charge fluctuations, see an insightful discussion by Girvin [17]). (b) Disorder will most likely not couple the left and right ends equally. Hence the boundary charge will not be exactly $e / 2$. There is as yet no conclusive experimental evidence for fractional charges in polyacetylene (but spin-charge separation was observed).

Consider Kitaev's toy model of one-dimensional $p$-wave superconductivity, which has relevance to topological superconductors [48]. Particle-hole symmetry plus bulk edge correspondence guarantees the presence of zero-energy modes. It exhibits a charge neutral particle that is divided between the two ends of the chain. These Majorana zero modes are expected to display non-Abelian statistics [49]. Figure 18 displays such zero modes of a finite length chain. (This is a well-known result and we show it here just for comparison with polyacetylene and ZGNRs.)

Now let us discuss edge states of a ZGNR. The probability density of the midgap states is fractionalized equally between

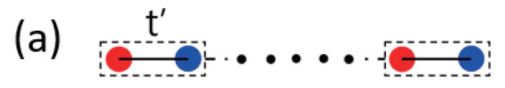

(b)

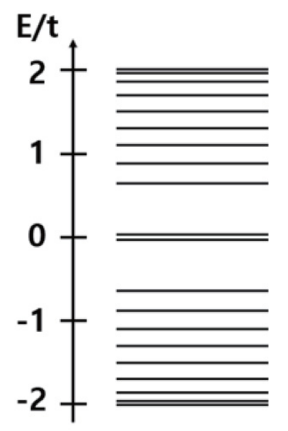

(c)

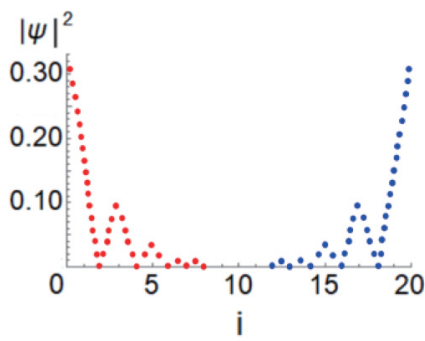

FIG. 17. (a) Finite-length dimer chain with unit cell containing two carbon atoms connected by single bond. The intracell hopping $t^{\prime}$ is smaller than the intercell hopping $t$. (b) Tight-binding energy spectrum. Two nearly degenerate gap states exist. (c) Probability density of a gap state as function of site index $i$. A peak is apparent at the red (blue) site at the left (right) end. The probability densities of the bonding and antibonding states are almost identical.

the left and right zigzag edges [9]. It is similar to fractionalization occurring at the end points of polyacetylene [15] and Kitaev's chain [46]. However, in interacting disordered ZGNRs fractional charges reside on the zigzag edges that form the side boundary of the ribbon, see Fig. 2. In addition, the presence of a gap $\Delta_{s}$ protects the fractional charges against quantum charge fluctuations.

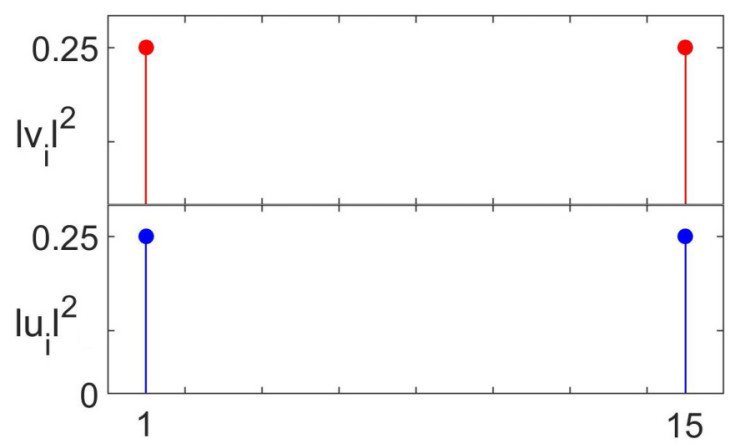

FIG. 18. Kitaev's chain has two degenerate zero-energy states. They can be combined to give one bonding and one antibonding states, see Fig. 7. For each of these states the probability to find an electron (hole) at site $i=1, \ldots, 15$ is $\left|u_{i}\right|^{2}\left(\left|v_{i}\right|^{2}\right)$. We have set the hopping parameter equal to the value of the gap $t=\Delta$ and the chemical potential $\mu=0$. A Majorana zero mode (half a real fermion mode) at each end of the chain is displayed. 
[1] K. S. Novoselov, A. K. Geim, S. V. Morozov, D. Jiang, M. I. Katsnelson, I. V. Grigorieva, S. V. Dubonos, and A. A. Firsov, Two-dimensional gas of massless Dirac fermions in graphene, Nature 438, 197 (2005).

[2] Y. Zhang, Y. W. Tan, H. L. Stormer, and P. Kim, Experimental observation of the quantum Hall effect and Berry's phase in graphene, Nature 438, 201 (2005).

[3] A. H. Castro Neto, F. Guinea, N. M. R. Peres, K. S. Novoselov, and A. K. Geim, The electronic properties of graphene, Rev. Mod. Phys. 81, 109 (2009).

[4] A good example of an SPT phase is polyacetylene. Chiral symmetry guarantees that end states exist.

[5] X.-G. Wen, Zoo of quantum-topological phases of matter, Rev. Mod. Phys. 89, 041004 (2017); Topological order: From longrange entangled quantum matter to a unified origin of light and electrons, ISRN Condens. Matter Phys. 2013, 198710 (2013).

[6] J. Wang, L. H. Santos, and X.-G. Wen, Bosonic anomalies, induced fractional quantum numbers, and degenerate zero modes: The anomalous edge physics of symmetry-protected topological states, Phys. Rev. B 91, 195134 (2015).

[7] S. Ryu and Y. Hatsugai, Topological Origin of Zero-Energy Edge States in Particle-Hole Symmetric Systems, Phys. Rev. Lett. 89, 077002 (2002).

[8] Y. H. Jeong and S.-R. Eric Yang, Topological end states and Zak phase of rectangular armchair ribbon, Ann. Phys. 385, 688 (2017). This paper shows that the Zak phase of a periodic armchair graphene nanoribbon is $0 \bmod 2 \pi$. However, it is not topologically trivial and the end charge has an integer value. Moreover, zigzag edges are unusual since their presence induces spin-splitting in the bulk.

[9] Y. H. Jeong, S. C. Kim, and S.-R. Eric Yang, Topological gap states of semiconducting armchair graphene ribbons, Phys. Rev. B 91, 205441 (2015). This paper demonstrates that no dimerization is needed to form a soliton in a carbon based system.

[10] M. Fujita, K. Wakabayashi, K. Nakada, and K. Kusakabe, Peculiar localized state at zigzag graphite edge, J. Phys. Soc. Jpn. 65, 1920 (1996).

[11] A ZGNR is a quasi-one-dimensional system and not one dimensional. A ribbon obviously has a width. Often properties of a quasi-one-dimensional system may be different from those of a strictly one-dimensional system.

[12] In an Anderson insulator disorder induces localization of electron states. In a Mott insulator the Fermi level is in the gap induced by many-body interactions. In a Mott-Anderson insulator both disorder and many-body interactions are important. Localized states often display spin-splitting.

[13] V. Dobrosavljevic, N. Trivedi, and J. M. Valles, Jr. (eds.) Conductor Insulator Quantum Phase Transitions (Oxford University Press, Oxford, 2012); D. Belitz and T. R. Kirkpatrick, The Anderson-Mott transition, Rev. Mod. Phys. 66, 261 (1994).

[14] Y. H. Jeong, S.-R. Eric Yang, and M.-C. Cha, Soliton fractional charge of disordered graphene nanoribbon, J. Phys.: Condens. Matter 31, 265601 (2019).

[15] W. P. Su, J. R. Schrieffer, and A. J. Heeger, Solitons in Polyacetylene, Phys. Rev. Lett. 42, 1698 (1979); A. J. Heeger, S. Kivelson, J. R. Schrieffer, and W. P. Su, Solitons in conducting polymers, Rev. Mod. Phys. 60, 781 (1988).

[16] S.-R. Eric Yang, Soliton fractional charges in graphene nanoribbon and polyacetylene: Similarities and differences,
Nanomaterials 9, 885 (2019). In this paper topological properties of polyacetylene and ZGNRs are compared.

[17] S. M. Girvin, in Topological Aspects of Low Dimensional Systems, edited by A. Comtet, T. Joliceur, S. Ouvry, and F. David (Springer-Verlag, Berlin, 2000).

[18] L. Yang, C. H. Park, Y. W. Son, M. L. Cohen, and S. G. Louie, Quasiparticle Energies and Band Gaps in Graphene Nanoribbons, Phys. Rev. Lett. 99, 186801 (2007).

[19] P. Ruffieux, S. Wang, B. Yang, C. Sanchez-Sanchez, J. Liu, T. Dienel, L. Talirz, P. Shinde, C. A. Pignedoli, and D. Passerone, On-surface synthesis of graphene nanoribbons with zigzag edge topology, Nature 531, 489 (2016).

[20] X.-G. Wen, Theory of the edge states in fractional quantum Hall effects, Int. J. Mod. Phys. B 6, 1711 (1992)

[21] C. de Chamon and E. Fradkin, Distinct universal conductances in tunneling to quantum Hall states: The role of contacts, Phys. Rev. B 56, 2012 (1997).

[22] M. Grayson, D. C. Tsui, L. N. Pfeiffer, K. W. West, and A. M. Chang, Continuum of Chiral Luttinger Liquids at the Fractional Quantum Hall Edge, Phys. Rev. Lett. 80, 1062 (1998).

[23] S. M. Girvin and K. Yang, Modern Condensed Matter Physics (Cambridge University Press, Cambridge, 2019).

[24] In an SPT phase symmetry determines properties of the system while in a topological ordered phase properties are beyond symmetry description.

[25] L. R. F. Lima, F. A. Pinheiro, R. B. Capaz, C. H. Lewenkopf, and E. R. Mucciolo, Effects of disorder range and electronic energy on the perfect transmission in graphene nanoribbons, Phys. Rev. B 86, 205111 (2012).

[26] T. Ando, Theory of electronic states and transport in carbon nanotubes, J. Phys. Soc. Jpn. 74, 777 (2005).

[27] S. C. Kim and S.-R. Eric Yang, Coulomb impurity problem of graphene in magnetic fields, Ann. Phys. 347, 21 (2014); S.-R. Eric Yang and H. C. Lee, X-ray edge problem of graphene, Phys. Rev. B 76, 245411 (2007).

[28] S.-R. Eric Yang and A. H. MacDonald, Coulomb Gaps in a Strong Magnetic Field, Phys. Rev. Lett. 70, 4110 (1993).

[29] S.-R. Eric Yang, A. H. MacDonald, and B. Huckestein, Interactions, Localization, and the Integer Quantum Hall Effect, Phys. Rev. Lett. 74, 3229 (1995).

[30] S.-R. Eric Yang, Z. Wang, and A. H. MacDonald, Thermodynamic and tunneling density of states of the integer quantum Hall critical state, Phys. Rev. B 65, 041302 (2001).

[31] L. Pisani, J. A. Chan, B. Montanari, and N. M. Harrison, Electronic structure and magnetic properties of graphitic ribbons, Phys. Rev. B 75, 064418 (2007).

[32] T. Stauber, P. Parida, M. Trushin M. V. Ulybyshev, D. L. Boyda, and J. Schliemann, Interacting Electrons in Graphene: Fermi Velocity Renormalization and Optical Response, Phys. Rev. Lett. 118, 266801 (2017).

[33] The number of zero modes in a ZGNR is proportional to the ribbon length. Zero modes are important in topological insulators because their presence may be related to ground-state degeneracy.

[34] An instanton is a nonperturbative effect. When it is applied to a double quantum well one finds that the energy splitting between the bonding and antibonding states is $\Delta E \propto e^{-1 / \lambda}$, which is 
singular in the coupling constant $\lambda$. In field theory an instanton is used to connect two vacua.

[35] S. C. Kim, P. S. Park, and S.-R. Eric Yang, States near Dirac points of a rectangular graphene dot in a magnetic field, Phys. Rev. B 81, 085432 (2010).

[36] When disorder is weak, van Hove singularities are present in the DOS, displaying a sharply peaked DOS near the gap edges. Because of these sharp peaks, even a weak disorder potential may generate numerous edge states that are fractionalized between the opposite zigzag edges.

[37] The gap of a disordered ZGNR is filled with localized states, whereas the states outside the gap are delocalized [14]. Thus, the usual one-dimensional localization theory does not apply to the Dirac electrons of GNRs $[3,26]$. A short-ranged disorder potential induces stronger localization of gap-edge states along the zigzag edges than a long-ranged disorder potential [25].

[38] H. C. Lee and S.-R. Eric Yang, Spin-charge separation in quantum Hall edge liquids, Phys. Rev. B 56, R15529(R) (1997).

[39] Spontaneously broken symmetry means: The Hamiltonian is invariant under spin rotation, but the ground state is not invariant.

[40] A. L. Efros and B. I. Shklovskii, Coulomb gap and low temperature conductivity of disordered systems, J. Phys. C: Solid State Phys. 8, L49 (1975).

[41] W. Kang, H. L. Stormer, L. N. Pfeiffer, K. W. Baldwin, and $\mathrm{K}$. W. West, Tunnelling between the edges of two lateral quantum Hall systems, Nature 403, 59 (2000); I. Yang, W. Kang, K. W. Baldwin, L. N. Pfeiffer, and K. W. West, Cascade of
Quantum Phase Transitions in Tunnel-Coupled Edge States, Phys. Rev. Lett. 92, 056802 (2004).

[42] R. de-Picciotto, M. Reznikov, M. Heiblum, V. Umansky, G. Bunin, and D. Mahalu, Direct observation of a fractional charge, Nature 389, 162 (1997); L. Saminadayar, D. C. Glattli, Y. Jin, and $\mathrm{B}$. Etienne, Observation of the $e / 3$ Fractionally Charged Laughlin Quasiparticle, Phys. Rev. Lett. 79, 2526 (1997).

[43] V. J. Goldman and B. Su, Resonant tunneling in the quantum Hall regime: Measurement of fractional charge, Science 267, 1010 (1995).

[44] Y. Yao, A. Liu, J. Bai, X. Zhang, and R. Wang, Electronic structures of silicene nanoribbons: Two-edge-chemistry modification and first-principles study, Nanoscale Res. Lett. 11, 371 (2016).

[45] V. Barone and J. E. Peralta, Magnetic boron nitride nanoribbons with tunable electronic properties, Nano Lett. 8, 2210 (2008).

[46] A. Y. Kitaev, Unpaired Majorana fermions in quantum wires, Phys.-Usp. 44, 131 (2001).

[47] In the Su-Schrieffer-Heeger effect half of soliton's spectral weight originates from the conduction band and the other half from the valence band.

[48] C. W. J. Beennakker, Search for Majorana fermions in superconductors, Annu. Rev. Con. Mat. Phys. 4, 113 (2013).

[49] A non-Abelian particle is neither a fermion nor a boson. On exchange of two non-Abelian particles their total wave function acquires a matrix Berry phase. 